\title{
Linear and nonlinear Rayleigh-Bénard-Marangoni instability with surface deformations
}

\author{
V. C. Regnier \\ CESAME, Unité de Mécanique Appliquée, Université Catholique de Louvain, Av. G. Lemaître 4, \\ B-1348 Louvain-La-Neuve, Belgium \\ P. C. Dauby and G. Lebon ${ }^{\text {a) }}$ \\ Université de Liège, Institut de Physique B5, B-4000 Liège 1, Belgium
}

(Received 11 June 1999; accepted 26 July 2000)

\begin{abstract}
Thermoconvective instabilities in a bilayer liquid-gas system with a deformed interface are investigated. In the first part of the work which is devoted to a linear approach, emphasis is put on the role of the upper gas layer on the instability phenomenon. The condition to be satisfied by the gas to remain purely conductive is established. The so-called Oberbeck-Boussinesq approximation is discussed and its range of validity is carefully defined. Instead of the classical Rayleigh, Marangoni, crispation, and Galileo numbers, new dimensionless groups are introduced. A critical comparison with several previous works is made. The nonlinear analysis consists in studying the different convective patterns which can appear above the threshold. Particular attention is devoted to the shape of the interface and the so-called "hybrid" relief. The amplitude of the deformation is also determined and comparison with experimental data is discussed. (C) 2000 American Institute of Physics. [S1070-6631(00)00911-9]
\end{abstract}

\section{INTRODUCTION}

Thermoconvective instabilities have been an important problem of fluid mechanics for many years. This general problem is well known and it is not necessary to present it in detail. ${ }^{1}$ Let us just recall that two different mechanisms are usually recognized as responsible for the appearance of motion in a fluid layer which is sufficiently heated from below. These mechanisms are gravity (Rayleigh-Bénard convection) and thermocapillarity (Bénard-Marangoni convection). When both mechanisms are active, the problem is usually referred to as the Rayleigh-Bénard-Marangoni instability. Thermocapillary effects are only present when the fluid has a free upper surface and is therefore in contact with an air layer. Under these circumstances, motion in the liquid can induce deformations of the liquid-gas interface.

Our analysis of surface deformations in the RayleighBénard-Marangoni instability is concerned with a linear and a nonlinear approach. In a first (linear) part, we carefully study the role of the air layer on the occurrence of thermoconvective instabilities in a two-layer system (see also Refs. 2 and 3). In the past, this problem has quite often been considered as a one-layer system wherein the air plays only a passive role. In the present work, we determine precisely under which conditions the air layer can actually be regarded as a "passive gas" and we derive in that case the corresponding boundary conditions at the top surface of the liquid. We show that the motion inside the gas phase can be disregarded provided its thickness is "smaller" than the thickness of the liquid layer. The gas can then be modeled as

\footnotetext{
a) Also at Louvain University, Unity TERM, B 1348 Louvain-La-Neuve, Belgium.
}

a purely conductive medium and a boundary condition for the temperature field in the liquid is derived in a rigorous way.

Another important issue is to examine the influence of surface deformations on the instability threshold. The problem has already been analyzed in several papers in the past. ${ }^{4-14}$ However, some of these works are not really quite general since the analysis is often restricted to particular fluids with well-defined thermophysical properties. Moreover, some of the reported results are not realistic from an experimental point of view or even are not consistent with the mathematical model used. These inconsistencies are related to the use of the Oberbeck-Boussinesq approximation, which is, for this reason, discussed in some detail. To delimit the domain of validity of the Boussinesq approximation, we introduce a new, somewhat unusual, nondimensional group which enables an easy checking of the consistency of the analysis. Moreover, other new dimensionless groups are also introduced in order to obtain results which are general and not valid only for some specific fluids.

In the nonlinear approach of the problem, we first examine the different possible convective patterns which can be observed above the linear threshold and compare our results with those for a flat interface ${ }^{15}$ and also with some experimental results by Eckert et al. ${ }^{16} \mathrm{We}$ also study the shape of the deformed interface in the nonlinear regime. We propose a theoretical description of the so-called "hybrid" relief (concave deformation within the convex relief) and determine the amplitude of the surface deflections. Eventually, our results are shown to be in satisfactory agreement with the experimental data reported by Cerisier et al. ${ }^{17}$

The paper is organized as follows. In Sec. II, the general mathematical model is briefly presented and the notation is 
introduced. The Oberbeck-Boussinesq approximation is discussed and a new nondimensional number is introduced to check the consistency of the model. In Sec. III, the thermomechanical coupling between the liquid and the gas layers is studied and the range of validity of the "conductive gas" hypothesis is established. To examine the influence of deformations on the instability threshold new nondimensonal groups are introduced and the general linear results are presented. Section IV is devoted to the nonlinear analysis of the system. In particular, we study the competition between the different convective patterns and determine the conditions to observe square cells, rolls, or hexagonal structures. The relief of the upper surface of the liquid is also studied and the height of the deformations is determined. Comparison with experimental data is discussed. Final comments and general conclusions are drawn in Sec. V.

\section{MATHEMATICAL MODEL}

The general equations and boundary conditions for thermoconvective instabilities in two superposed immiscible fluids are well known ${ }^{18}$ and will not be recalled here. Only some comments on the general hypotheses underlying the model will be formulated and the notation will be defined.

The two-fluid system consists of two horizontally infinite immiscible layers of liquid and gas which are enclosed between two solid and perfectly heat conducting boundaries. The thicknesses of these layers are, respectively, $d$ and $d_{\text {gas }}$ $=d d_{r}\left(d_{r}\right.$ is the ratio of the thicknesses of the gas and the liquid layers). The system is heated from below. The analysis is carried out in the frame of the well-known "simplified Oberbeck-Boussinesq model," 19 which enables one to deduce equations for the perturbations with respect to the conductive solution (for simplicity, these equations are sometimes referred to as the "Boussinesq equations"). Moreover, the surface deformations are assumed to remain small so that all equations and boundary conditions are limited to firstorder erms in the surface deflection.

In this work, we use Cartesian coordinates with origin located on the nondeformed liquid-gas interface and the $z$ axis pointing in the opposite direction of gravity. The equations are written in nondimensional form with distances scaled by $d$. The bottom of liquid layer is located at $z$ $=-1$ while the top of the gas phase is at $z=d_{r}$. The nondimensional deflection of the liquid-gas interface will be denoted $\eta$. The time scale is given by $d^{2} / \kappa$, with $\kappa$ the heat diffusivity of the liquid. The temperature scale is chosen as $\beta d$, with $\beta$ the vertical temperature gradient within the liquid layer in a purely conductive state, and the velocity is scaled by $\kappa / d$. The symbols $\mathbf{u}=(u, v, w), p$, and $T$ represent the nondimensional velocity, pressure, and temperature perturbed fields in the lower liquid while the same symbols with index gas represent the corresponding quantities in the upper gas; $\alpha_{r}$ is the ratio of the coefficients of thermal expansion of the gas and the liquid; $\mu_{r}, \kappa_{r}$, and $k_{r}$, are similarly defined as the ratios of dynamic viscosities, heat diffusivities, and heat conductivities, respectively; $\rho_{r}$ is the ratio of the densities of the gas and the liquid when these densities are considered at a common reference temperature, which is chosen as $T_{0}$, the temperature of the gas-liquid interface in a purely conductive state. The Rayleigh and Prandtl numbers Ra and Pr are given by

$$
\begin{aligned}
& \operatorname{Pr}=\frac{\nu}{\kappa}, \\
& \operatorname{Ra}=\frac{g \alpha \beta d^{4}}{\nu \kappa},
\end{aligned}
$$

where $\nu$ and $\alpha$ are the liquid kinematic viscosity and coefficient of thermal expansion.

Although the simplified Boussinesq model is widely used in the literature on thermoconvective instabilities, it is worth recalling briefly its range of validity. Indeed, some published results based on this model are incompatible with the basic assumptions of the model itself (see Sec. III B 1). For the Boussinesq equations to be valid, it is required that the temperature differences within the system remain small. When $\Delta T$ becomes too large, non-Boussinesquian effects can appear. These effects have been studied elsewhere ${ }^{20,21}$ and will not be considered here.

In order to quantify the "smallness" of the temperature differences within the system we introduce the following nondimensional number, which is assumed to remain smaller than 1:

$$
\delta_{\rho}=\alpha \beta d \ll 1 .
$$

This nondimensional quantity is proportional to the temperature drop across the liquid layer and is related to the gravitational instability mechanism through the coefficient of thermal expansion $\alpha$. In the following, this parameter will be considered as the main control parameter describing the external forcing on the system.

If condition (2.3) is satisfied, the Boussinesq model may be considered as valid in the fluid (see, e.g., Ref. 18, p. 5). Similarly, the same model is applicable to describe the gas phase provided $\delta_{\rho_{\text {gas }}}=\alpha_{\text {gas }} \beta_{\text {gas }} d_{\text {gas }} \ll 1$. For $d_{r}$ of order 1 , it is seen from Table I that the ratio $\delta_{\rho_{\mathrm{gas}}} / \delta_{\rho}=\left(\alpha_{r} / k_{r}\right) d_{r}$ is larger than 1 for all liquid-air systems. Nevertheless, for all liquids but water and glycerin, this quantity keeps a reasonable value when $d_{r}$ is (much) smaller than 1 . We will therefore also use the Boussinesq equations in the gas, even if the approximation is less satisfactory than in the liquid.

The boundary conditions for our problem are the following. It is assumed that the temperature is fixed and that the velocity vanishes at the bottom of the liquid and at the top of the gas. The deflection $\eta$ of the interface obeys the usual kinematic condition. The temperature, heat flux, and velocity fields are continuous across the interface while the mechanical stresses are supposed to be in equilibrium at the interface. The complete mathematical expressions of these boundary conditions can be found for instance in Ref. 18 and depend on the Galileo, crispation, and Marangoni dimensionless numbers $G, \mathrm{Cr}$, and $\mathrm{Ma}$, defined as

$$
G=\frac{\rho_{0}\left(1-\rho_{g}\right) g d^{3}}{\mu \kappa},
$$


TABLE I. Ratios between some thermophysical properties of different liquids and the corresponding properties of air ("Sil." is written for silicone and "St" is written for Stokes).

\begin{tabular}{lcccccc}
\hline \hline & $\rho_{r}\left(10^{-3}\right)$ & $\mu_{r}\left(10^{-3}\right)$ & $\kappa_{r}$ & $k_{r}$ & $\alpha_{r}$ & $\alpha_{r} k_{r}^{-1}$ \\
\hline Water & 1.2 & 18.2 & 112 & 0.0285 & 20.2 & 709 \\
Glycerin & 0.952 & 0.0194 & 180 & 0.0592 & 6.80 & 115 \\
Sil. 47V 0.1St & 1.29 & 1.96 & 215 & 0.131 & 3.15 & 24.0 \\
Sil. 47V 1St & 1.24 & 0.184 & 141 & 0.106 & 3.60 & 34.0 \\
Sil. 47V 10St & 1.24 & 0.0188 & 142 & 0.106 & 3.60 & 34.0 \\
Sil. DC200 1St & 1.24 & 0.190 & 145 & 0.113 & 3.50 & 31.0 \\
Benzene & 1.37 & 28.3 & 160 & 0.113 & 2.81 & 24.9 \\
Toluene & 1.39 & 31.0 & 144 & 0.106 & 3.21 & 30.3 \\
Acetone & 1.69 & 611 & 162 & 0.105 & 2.33 & 22.2 \\
Ethanol & 1.52 & 153 & 179 & 0.102 & 3.18 & 31.2 \\
\hline \hline
\end{tabular}

$$
\begin{aligned}
& \mathrm{Cr}=\frac{\mu \kappa}{\sigma_{0} d}, \\
& \mathrm{Ma}=\frac{\gamma \beta d^{2}}{\mu \kappa} .
\end{aligned}
$$

The subscript zero indicates that the corresponding quantity is taken at temperature $T_{0}$. The quantity $\gamma$ which appears in the definition of the Marangoni number is introduced by approximating the variations of the surface tension at the liquid-gas interface by a linear function of $T$,

$$
\sigma(T)=\sigma_{0}-\gamma\left(T-T_{0}\right),
$$

where $\gamma$ is positive for most liquids and can be considered as a constant. This linear law can be considered as valid if the temperature variations inside the system remain small. This condition can be made explicit by introducing the following nondimensional group:

$$
\delta_{\sigma}=\frac{\gamma \beta d}{\sigma_{0}} .
$$

In parallel with $\delta_{\rho}$ defined in (2.3), $\delta_{\sigma}$ is proportional to the temperature drop across the liquid layer but this new number is related to the thermocapillary instability mechanism. The ratio of these two numbers is denoted

$$
A=\frac{\delta_{\sigma}}{\delta_{\rho}}=\frac{\gamma}{\sigma_{0} \alpha}
$$

and is more or less constant and of order 1 for many liquids, as shown in Table II. In the following we will consider $\delta_{\rho}$ as

TABLE II. Values of the dimensionless numbers $A, d_{r}^{\text {crit }}$ for some fluids.

\begin{tabular}{lcl}
\hline \hline & $A$ & $d_{r}^{\text {crit }}$ \\
\hline Water & 12.2 & 1.57 \\
Glycerin & 10.4 & 0.534 \\
Silicone 47V 0.1St & 3.22 & 2.43 \\
Silicone 47V 1St & 3.54 & 1.13 \\
Silicone 47V 10St & 3.83 & 0.64 \\
Silicone DC200 1St & 2.90 & 1.16 \\
Benzene & 3.83 & 4.29 \\
Toluene & 4.00 & 4.07 \\
Acetone & 5.83 & 4.80 \\
Ethanol & 3.69 & 3.48 \\
\hline \hline
\end{tabular}

the instability control parameter, while $A$ will be kept fixed to a typical value of the order of one.

The normal stress condition at the interface deserves special attention. This condition is given by

$$
\left(p-p_{\text {gas }}\right)-G \eta+\mathrm{Cr}^{-1} \mathcal{K}=\mathbf{n} \cdot\left(\tau-\mu_{r} \tau_{\text {gas }}\right) \cdot \mathbf{n},
$$

where $\mathcal{K}$ is the curvature of the interface while $\mathbf{n}$ is the normal unit vector. The quantity $\tau$ is given by $\tau=(\boldsymbol{\nabla u}$ $\left.+(\boldsymbol{\nabla u})^{T}\right)$, with a similar expression for $\tau_{\text {gas }}$.

It is worth recalling that this relation is not valid in general but only when $\delta_{\rho}$ and $\delta_{\sigma}$ remain small ${ }^{18}$ (otherwise, other terms are present).

To summarize, let us recall briefly the context of our analysis. First of all, it is assumed that the temperature differences remain small across the system $\left(\delta_{\rho} \ll 1\right)$, which ensures that the Boussinesq hypotheses are met. The smallness of $\delta_{\rho}$ also justifies the linear dependence of surface tension with respect to $T$ as well as the simplified form (2.10) of the normal stress condition. Also keep in mind that throughout the analysis, surface deflections are assumed to remain small.

\section{LINEAR STABILITY ANALYSIS}

The linear stability analysis is carried out by using a normal modes method. ${ }^{22}$ The wave number and the growth rate of the perturbations are denoted $k$ and $s$, respectively. The stability equations are reduced in a standard way to a system of ordinary differential equations in $z$ for the vertical velocity amplitude $W(z)$, the temperature amplitude $\Theta(z)$, and vertical vorticity amplitude $\Omega(z)$. The equation for the vertical vorticity is uncoupled from these for $W(z)$ and $\Theta(z)$ and an analytical solution ${ }^{22,23}$ shows that the vorticity perturbations are unconditionally stable.

The eigenvalue problem for the vertical velocity and the temperature must be solved numerically. In the present work, a spectral Chebyshev-Tau method is used to discretize the equations. As a first general result, let us mention that the threshold is never oscillatory. Therefore only stationary convection will be investigated, with either a zero or a nonvanishing wave number. 
TABLE III. Thermophysical properties of air and of silicone oil DC200.

\begin{tabular}{lcc}
\hline \hline & Air & Silicone DC200 1St \\
\hline$\rho\left(10^{3} \mathrm{~kg} / \mathrm{m}^{3}\right)$ & 0.00120 & 0.968 \\
$\nu\left(10^{-4} \mathrm{~m}^{2} / \mathrm{s}\right)$ & 0.152 & 1.00 \\
$\kappa\left(10^{-7} \mathrm{~m}^{2} / \mathrm{s}\right)$ & 160 & 1.10 \\
$k^{T}(\mathrm{~W} / \mathrm{mK})$ & 0.017 & 0.150 \\
$\alpha^{T}\left(10^{-4} \mathrm{~K}^{-1}\right)$ & 34.0 & 9.60 \\
$\sigma\left(10^{-2} \mathrm{~N} / \mathrm{m}\right)$ & $\cdots$ & 2.09 \\
$\gamma\left(10^{-4} \mathrm{~N} / \mathrm{mK}\right)$ & $\cdots$ & -0.58 \\
\hline \hline
\end{tabular}

\section{A. Influence of the convection in the gas layer on the instability and reduction to a one-layer problem}

In this section, we examine how far convection in the gas influences the instability mechanism. We show that in most experimental situations the gas can be regarded as mechanically passive, which means that the motion in the gas can be neglected. Our problem is thus different from that considered by Rasenat et al., ${ }^{2}$ where a bilayer system with both layers close to instability was investigated. More precisely it will be proved that, for both the finite and infinite wavelength instabilities, the two layers can be considered as mechanically uncoupled due to the smallness of $\mu_{r}$, the ratio of the dynamic viscosities of the gas and of the liquid, but in contrast the two layers remain thermally coupled.

First, let us study the coupling between the two layers for the finite wavelength instability, and consider the particular case of an air-silicone DC200 bilayer system. ${ }^{24}$

The thermophysical properties of these two fluids are given in Table III. The thickness of the oil layer is fixed to $d=2 \mathrm{~mm}$ and the thickness of the gas layer is allowed to vary. The critical Rayleigh number and the corresponding critical Rayleigh number in the gas, defined as $\mathrm{Ra}_{\text {gas }}$ $=\rho_{0 \mathrm{gas}} \alpha_{\mathrm{gas}} \beta_{\mathrm{gas}} d_{\mathrm{gas}}^{4} /\left(\mu_{\mathrm{gas}} \kappa_{\mathrm{gas}}\right)$, are represented in Fig. 1 as functions of the nondimensional thickness of the gas $d_{r}$. The values of $\delta_{\rho}$ in the liquid, $\delta_{\rho}$ in the gas, and $\delta_{\sigma}$ can be

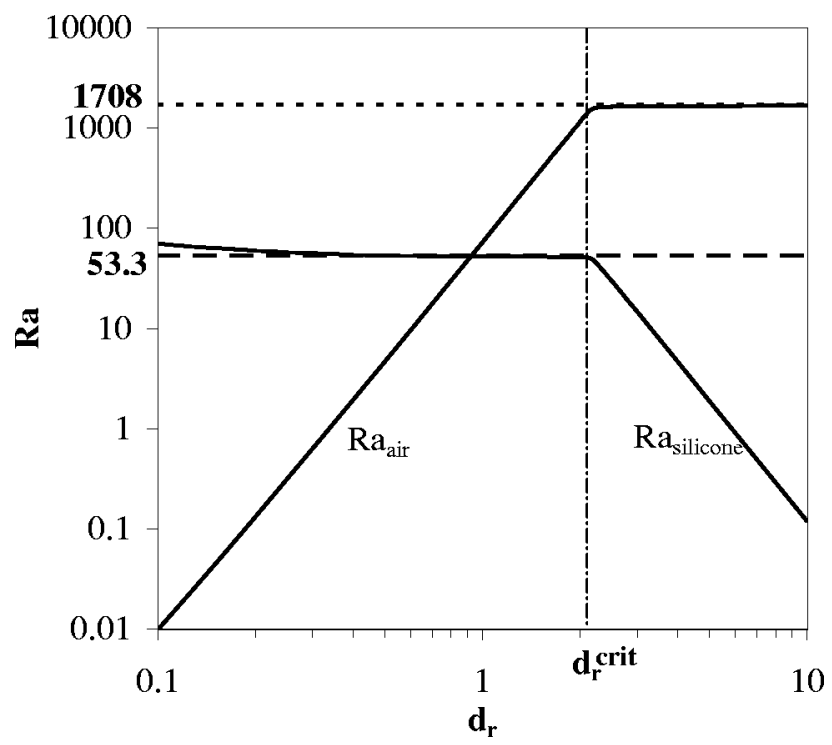

FIG. 1. Critical Rayleigh number vs the dimensionless thickness $d_{r}$ in a silicon-air bilayer system $(d=2 \mathrm{~mm})$. The small and large dashes correspond to $\mathrm{Ra}=1708$ and 53.3 , respectively. deduced from the values of the Rayleigh numbers and shown to remain small. As explained previously, the smallness of these $\delta$ 's confirms the consistency of the model.

A noticeable result in Fig. 1 is the existence of a critical relative thickness $d_{r}^{\text {crit }}$ below which the critical Rayleigh number (in the liquid) is more or less constant and above which the critical Rayleigh number in the gas is also nearly constant. This sudden change in the behavior of the system when $d_{r}$ is varied indicates a drastic change in the physical mechanism giving rise to the instability.

For $d_{r}$ larger than the threshold value $d_{r}^{\text {crit }}$, the Rayleigh number in the gas is close to 1708 , which is the well-known value for a one-layer system with two rigid and perfectly heat conducting boundaries, ${ }^{25,26}$ in Fig. 1, this value of the Rayleigh number is represented by a horizontal line with small dashes. A detailed analysis of the slopes of the curves in Fig. 1 also shows that, for $d_{r}>d_{r}^{\text {crit }}$, the critical Rayleigh number (in the liquid) decreases as $d_{r}^{-4}$, which is expected since $\mathrm{Ra}_{\text {gas }}=\operatorname{Ra} \rho_{r} \alpha_{r} d_{r}^{4} /\left(\mu_{r} \kappa_{r} k_{r}\right)$ with $\mathrm{Ra}_{\text {gas }}$ more or less constant. This behavior means that for a large $d_{r}$, the upper phase behaves more or less as if the liquid was a perfectly heat conducting solid wall: The liquid does not play any active role in the instability, which thus takes place in the gas only. This result is easily understood because the heat conductivity of the liquid is much larger (about 10 times) than that of the gas and also because the viscosity of the liquid is much larger than that of the gas $\left(\mu_{r}=10^{-4}\right)$. Note also that the Marangoni condition at the bottom of the gas layer does not influence the instability because the liquid behaves as a good heat conductor and therefore dampens the horizontal variations of temperature. For such large values of $d_{r}$, the instability in the gas is thus completely, i.e., mechanically and thermally, uncoupled from the lower liquid layer.

In contrast, when $d_{r}$ is smaller than $d_{r}^{\text {crit }}$, the instability mechanism takes place mainly in the liquid phase. This situation is the most interesting one for us since we are mainly interested in instability phenomena within the liquid. In this case, the critical Rayleigh number is close to the value derived from Nield's formula ${ }^{26} \quad \mathrm{Ra}_{c}^{\text {Nield }} \simeq\left((669)^{-1}\right.$ $\left.+(79.6 \Gamma)^{-1}\right)^{-1}$ with the parameter $\Gamma$ defined as the ratio of the Rayleigh and Marangoni numbers:

$$
\Gamma=\frac{\mathrm{Ra}}{\mathrm{Ma}}=\frac{\rho_{0} g \alpha}{\gamma} d^{2} .
$$

This critical Rayleigh number refers to a one-layer system with an upper adiabatic free surface and a perfectly heat conducting rigid bottom wall. In our problem, this Rayleigh number is equal to 53.3 and is plotted in Fig. 1 with long dashes. It should be observed that the critical Rayleigh number (in the liquid), although close to Nield's result, is not exactly equal to the latter. Moreover, $\mathrm{Ra}_{c}$ varies slightly with $d_{r}$. This means that the interface cannot be considered as an actual free adiabatic boundary and that the gas is not completely passive.

Note that the above-mentioned study was performed for the particular case of an air-silicone oil system, with a fixed oil depth. We have checked that the same conclusion remains true for other classes of fluids (see also Ref. 3), and 


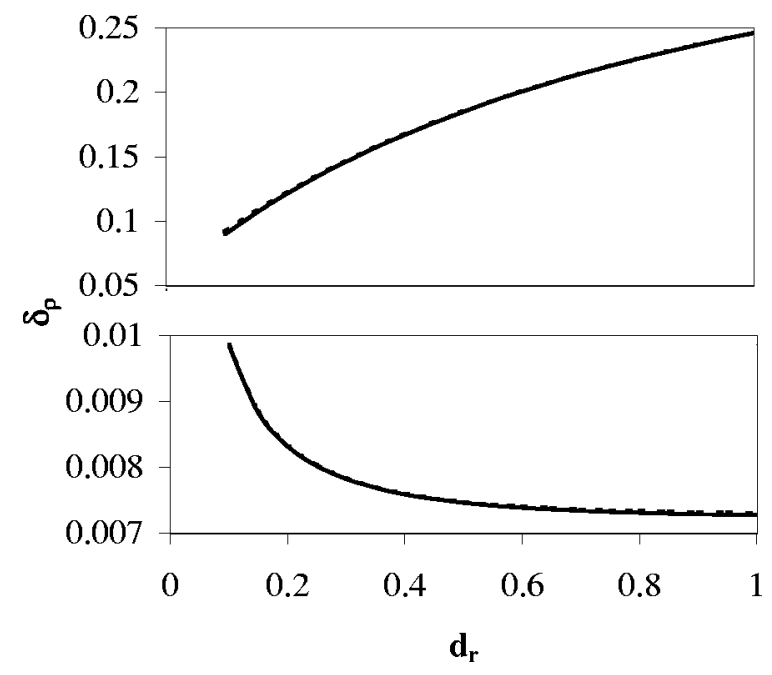

FIG. 2. Critical $\delta_{\rho}$ vs $d_{r}$ for the air-silicone oil DC200 system. Continuous lines correspond to the complete bilayer analysis and while dashed lines refer to the simplified approach with the motion of the gas neglected. The upper and lower pictures refer to the infinite and finite wave-number modes, respectively.

that the critical value $d_{r}^{\text {crit }}$ is still found to correspond to a Rayleigh number in the gas equal to 1708 . For many gasliquid (Table II) systems it is easy to check that $d_{r}^{\text {crit }}$ is of the order of unity. Since we are concerned with the study of instabilities within the liquid, we will always take in the following an air depth smaller than that of the liquid.

When the instability mechanism takes place mainly in the liquid phase, we have seen that the thickness of the gas layer may nevertheless slightly influence the appearance of convection in the liquid. We prove now that only thermal transport in the gas phase is important and that the motion of the upper layer can systematically be neglected.

First, we note that the rather small value of $\mu_{r}$ prevents any mechanical influence of the gas on the liquid. Moreover, the motion of the gas does not modify greatly the temperature profile within the gas phase. Consider indeed the (thermal) Péclet number Pe, which is a measure of the ratio of the time scales for thermal diffusion and thermal convection. In the gas, the Péclet number is given by $U_{\text {gas }} d_{\text {gas }} / \kappa_{\text {gas }}$, where $U_{\text {gas }}$ is a representative velocity scale in the gas. Since velocity is continuous across the liquid-gas interface, $U_{\text {gas }}$ is of the same order as the representative velocity scale in the liquid, which we chose as $\kappa / d$, the velocity of thermal perturbations in a diffusive state. An estimation of the Péclet number in the gas is thus $\mathrm{Pe}_{\mathrm{gas}}=d_{r} / \kappa_{r}$. Since $\kappa_{g}$ is of order $10^{2}$ for many liquid-gas systems, the Péclet number in the gas remains rather small if $d_{r}$ is of order 1 . As a consequence, the linear temperature profile in the gas will only undergo minute modifications due to the gas motion, which can therefore be disregarded in the analysis of the instability of the liquid.

A quantitative analysis of the influence of the motion of the gas is summarized in Fig. 2. In this picture, the critical value of $\delta_{\rho}$ for the air-silicone oil system is represented for both the complete bilayer analysis and for the simplified approach with the motion of the gas neglected. It is clear that the motion inside the upper layer can safely be neglected for $d_{r}<1$. It can also be concluded that the weak dependence of $\mathrm{Ra}_{c}$ with respect to $d_{r}$ in Fig. 1 originates only from thermal effects in the gas layer.

Consider now the zero wave-number instability. ${ }^{27}$ An analytical asymptotic analysis of the stability problem can be carried out in this case. ${ }^{28}$ The result for the air-silicone oil system is represented in Fig. 2, which clearly shows that the threshold is practically not changed by disregarding the motion in the gas phase, provided $d_{r}$ is not very small.

Provided $d_{r}$ is neither too small nor larger than about 1 , the motion in the gas phase can thus be neglected in the study of the instabilities in the lower liquid and the "conductive gas" hypothesis is valid. The exact solution for heat conduction in the upper layer can be used to derive the thermal boundary condition at the gas-liquid interface. This procedure was already followed in Refs. 29 and 30 for a flat interface and results in a "Biot condition" for the temperature field in the liquid. For a deformable interface, this equation is written as ${ }^{23}$

$$
D \Theta+\mathrm{Bi}_{r}(k) \Theta=\left(1-k_{r}^{-1}\right) \mathrm{Bi}_{r}(k) H \quad \text { at } z=0,
$$

with a Biot number given by $\mathrm{Bi}_{r}(k)=k_{r} k / \tanh k d_{r}$. All the results given in the following are based on this reduction of the system to a single-layer system for which the sole thermal influence of the gas on the liquid instability is taken into account by introducing the Biot boundary condition (3.2). It is also worth stressing that this conductive gas hypothesis is usually taken for granted, both in experimental (Refs. 24 and 31 ) and theoretical (Refs. 4, 7, 11-13, 26, 32-36, and 30) works. To our knowledge, however, the present paper provides the first complete justification and limitations of this important assumption.

\section{B. Influence of the interface deformation}

An exhaustive study of thermoconvective instabilities in a liquid layer with a deformable upper surface is not a straightforward task owing to the rather large number of parameters. When the motion of the gas is neglected, the problem still depends on seven independent quantities which are often chosen as $\operatorname{Pr}, \mathrm{Ra}, \mathrm{Ma}, \mathrm{Cr}, G, d_{r}$, and $k_{r}$. As our analysis has shown that the instability is never oscillatory, the Prandtl number can be dropped and six parameters are left. Besides the thermophysical properties of the fluids, these six quantities depend on the temperature drop between the bottom and the top of the system, which is the main experimental control parameter, and also on the thicknesses of the gas and liquid layers, which can also easily be varied in experiments.

To analyze a problem depending on so "many" parameters, two different ways are open. The simplest one consists in selecting two particular fluids. All the thermophysical properties are then fixed and the only variable parameters are the thicknesses of the two layers. The drawback of such a methodology is the lack of generality. In the second approach, which is followed in the present paper, the results are expressed as functions of one single among the six parameters, the others being kept fixed. It is important to realize, 
however, that this kind of approach can lead to dramatic inconsistencies when the fixed and variable parameters are not selected with care, as shown in the following.

\section{Comments on previous works}

The influence of deformations of the upper surface on thermoconvective instabilities has been studied by several authors. ${ }^{4-14}$ Some of these works are now briefly commented on. The first analysis was carried out in 1964 by Scriven and Sterling. ${ }^{4}$ They showed that, for the pure thermocapillary problem, convection sets in with a zero wave number and a zero value of the Marangoni number. Their results were revisisted in 1966 by Smith, ${ }^{5}$ who was able to obtain a nonzero value of the critical Marangoni number by including gravity waves at the free surface. The coupled thermogravitational and thermocapillary instability was studied by Zeren and Reynolds ${ }^{6}$ in the particular case of a water-benzene system. The one-layer thermocapillary instability was investigated by Takashima in 1981. ${ }^{7,8}$ Among other results, this author showed that oscillatory convection was possible for negative values of the Marangoni number, which corresponds either to a fluid layer heated from above, or to a fluid heated from below but whose surface tension increases with temperature. Unfortunately, some of Takashima's results are not consistent with Boussinesq's assumption which was used to derive the model equations. Indeed, although the condition $\delta_{\sigma}<1$ is satisfied as far as stationary instabilities are concerned, this relation is clearly violated in the analysis of oscillatory convection for Bond numbers smaller than $10^{-1}(\mathrm{Bo}=G \mathrm{Cr})$. Moreover, it is important to stress that the selection between the variable and fixed parameters can lead to misinterpretations of the results. In Takashima's papers, the fluid layers have a thickness varying between 0.1 and $1 \mathrm{~mm}$ and the results are given for fixed values of $\mathrm{Bo}, \mathrm{Cr}$, and $\mathrm{Pr}$, between which the following relation holds: $\mathrm{CrBo}^{-1} \mathrm{Pr}^{-1}$ $=\kappa^{2} g^{-1} d^{-3}$. Since $\kappa$ is more or less constant for most liquids, the above-mentioned result imposes strong restrictions on the values of the thickness and the nondimensional numbers. It is easy to check that some of Takashima's results with fixed $\mathrm{Bo}, \mathrm{Cr}$, and $\mathrm{Pr}$ correspond to values of $d$ which are outside the range initially proposed. For this reason, the possibility for two simultaneously unstable oscillatory convective modes with different wave numbers and frequencies is not realistic. Similar comments can be repeated about the works by Gouesbet et al. ${ }^{12}$ and by Pérez and Carneiro. ${ }^{13}$ These authors complement the analyses of Takashima by taking the gravity instability mechanism into account. However, most of their results do not satisfy condition (2.3), which is a necessary condition for the Boussinesq equations as well as the simplified boundary condition (2.10) to be valid. In addition, in Ref. 13, the selection of the variable and fixed nondimensional numbers is also questionable. When gravity effects are taken into account in the instability analysis, the crispation, Bond, Prandtl, and $\Gamma$ numbers verify $\mathrm{Cr}|\Gamma|^{-1} \mathrm{Bo}^{-1} \operatorname{Pr}^{-1}=\kappa^{2} \rho \alpha|\gamma d|^{-1}$. With typical values (SI units) $\kappa=10^{-7}, \rho=10^{3}, \quad \alpha=10^{-3}$, and $|\gamma|=10^{-4}$, the thickness of the liquid layer is given by $d$ $=10^{-11}|\Gamma|^{-1} \mathrm{Cr}^{-1}$. This means that for $\mathrm{Cr}=10^{-3}$, the simultaneous occurrence of a stationary and an oscillatory mode of convection corresponds to the unrealistic value of $d=3.10^{-6} \mathrm{~mm}$ when $|\Gamma| \simeq 33$. Similarly, for $|\Gamma| \simeq 0.1$, two oscillatory modes are simultaneously unstable only in a liquid layer with the unrealistic thickness of $10^{-3} \mathrm{~mm}$. To close this section, we would like to mention an interesting paper by Goussis and Kelly ${ }^{11}$ which is quite similar to ours as they also introduce nonconventional dimensionless numbers. However, unlike our paper, their analysis is restricted to pure thermocapillary convection.

\section{New nondimensional groups}

A way out to avoid the difficulties and problems raised previously is to introduce new dimensionless numbers. The reasons for introducing these new nondimensional groups are twofold: first to make them more directly accessible to experiments and second, to allow an easier checking of the consistency of the mathematical model.

We first recall that the properties of the "purely conductive gas" are completely specified by the knowledge of the two-dimensional quantities $d_{\text {gas }}$ and $k_{\text {gas }}$. For the lower liquid layer, we have already mentioned that the temperature drop across the system and the thickness of the layer are the only quantities which can be varied independently, easily, and continuously in experiments. Unfortunately, these dimensional quantities appear in the definition of several "classical" nondimensional numbers (to be explicit, they are present in $\mathrm{Ra}, \mathrm{Ma}, \mathrm{Cr}$, and $G$ ), which makes the analysis of the influence of each of these parameters rather cumbersome. Therefore, it should be preferable to combine the "classical" nondimensional numbers so that both the thickness $d$ of the liquid layer and the temperature difference $\Delta T$ across the liquid appear in one single well-defined dimensionless number.

As a nondimensional version for $d$ we select $\Gamma$ $=\mathrm{Ra} / \mathrm{Ma}$ defined by (3.1) and already introduced in several other works (e.g., Refs. 6 and 13).

For the temperature drop across the liquid, we suggest taking $\delta_{\rho}$, given in (2.3), as a new nondimensional group. This choice is dictated by the fact that condition (2.3) allows one to directly check the consistency of the analysis with the Boussinesq approximation.

The last two nondimensional numbers are the parameter $A$ defined in (2.9) and the parameter $N$ given by

$$
N=\frac{1}{G}\left(\frac{\mathrm{Ra}}{\mathrm{Ma}}\right)^{3 / 2}=\nu \kappa\left(\rho^{3} g \frac{\alpha^{3}}{\gamma^{3}}\right)^{1 / 2} .
$$

These quantities are introduced because $A$ is the sole nondimensional parameter which contains the surface tension $\sigma_{0}$ while the kinematic viscosity $\nu$ appears only in $N$. Moreover, the value of parameter $A$ remains about constant for a wide variety of fluids and can therefore be assigned a fixed value without loss of generality. The influence of viscosity on the stability problem is thoroughly described by the parameter $N$. Since the viscosity of silicone oils can be changed more or less continuously by mixing oils of different viscosities, while the other thermophysical properties remain unvaried, this parameter $N$ is very useful for compari- 
TABLE IV. Values of the quantities $N, N / \nu$, and $F$ for some fluids; SI units are used for $\nu$.

\begin{tabular}{lccc}
\hline \hline & $N$ & $N / \nu$ & $F$ \\
\hline Water & $1.68 \times 10^{-8}$ & 0.0168 & $4.2 \times 10^{8}$ \\
Glycerin & $7.01 \times 10^{-5}$ & 0.094 & $1.2 \times 10^{8}$ \\
Silicone 47V 0.1St & $6.63 \times 10^{-6}$ & 0.663 & $2.03 \times 10^{7}$ \\
Silicone 47V 1St & $8.76 \times 10^{-5}$ & 0.876 & $1.00 \times 10^{7}$ \\
Silicone 47V 10St & $8.78 \times 10^{-4}$ & 0.878 & $1.01 \times 10^{7}$ \\
Silicone DC200 1St & $6.99 \times 10^{-5}$ & 0.699 & $1.38 \times 10^{7}$ \\
Benzene & $1.64 \times 10^{-7}$ & 0.223 & $4.51 \times 10^{7}$ \\
Toluene & $1.65 \times 10^{-7}$ & 0.242 & $3.70 \times 10^{7}$ \\
Acetone & $5.80 \times 10^{-8}$ & 0.138 & $7.24 \times 10^{7}$ \\
Ethanol & $4.69 \times 10^{-7}$ & 0.310 & $3.60 \times 10^{6}$ \\
\hline \hline
\end{tabular}

son with experiments. Moreover, it follows from Table IV that the ratio $N / \nu$ keeps a more or less constant order of magnitude for many fluids, which reinforces the selection of $N$ as a relevant nondimensional expression of viscosity.

To summarize, we propose using the following set of dimensionless numbers, namely $\Gamma$ (thickness), $\delta_{\rho}$ (temperature drop), $A$ (surface tension), and $N$ (viscosity). In terms of these new quantities, the "classical," Rayleigh, Marangoni, crispation, and Galileo nondimensional numbers are written as

$$
\begin{aligned}
& \mathrm{Ra}=\delta_{\rho} \Gamma^{3 / 2} N^{-1}, \quad \mathrm{Ma}=\delta_{\rho} \Gamma^{1 / 2} N^{-1}, \\
& \mathrm{Cr}=A \Gamma^{-1 / 2} N, \quad G=\Gamma^{3 / 2} N^{-1} .
\end{aligned}
$$

\section{Linear results}

Previous analyses have clearly shown that convection may set in under three different forms. First, the instability can be stationary, with a nonzero wave number. This kind of instability is similar to what is observed in a one-layer system with a flat upper free surface. When the interface between both fluids is deformed, instabilities can also appear either as a steady convection with a zero horizontal wave number or as an oscillatory motion.

The results presented here are restricted to experimental situations in which the upper gas may be considered as mechanically passive. As explained before, this is achieved by assuming $d_{r}$ smaller than about one. In the following we take $d_{r}=0.5$. When the upper gas is air, we are allowed to take $k_{r}=0.1$, which is a typical value for many liquid-air cells as seen from Table I. Similarly, the parameter $A$ is taken equal to 5 , which is a reasonable value for many usual fluids. The final results will thus depend on the thickness of the layer and on the viscosity of the liquid through parameters $\Gamma$ and $N$, respectively.

A first general conclusion of our analysis is the steady character of the linear threshold in air-liquid systems heated from below. Oscillatory convection cannot be observed under realistic experimental conditions for which the Boussinesq equations are valid $\left(\delta_{\rho c} \ll 1\right)$. For this reason the value of the Prandtl number will not influence the linear results.

The main results of our study are summarized in Fig. 3, where the critical nondimensional temperature difference across the system is plotted, for different values of the viscosity parameter $N$, as a function of $\Gamma$. Since $\alpha$ is more or

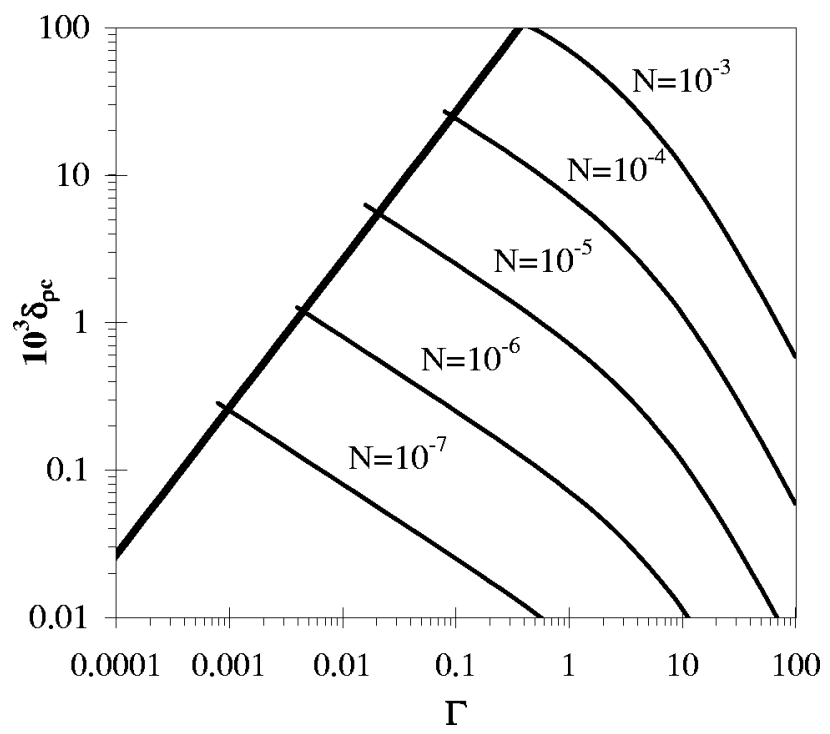

FIG. 3. Critical $\delta_{\rho}$ vs $\Gamma$ for different values of $N\left(A=5, d_{r}=0.5, k_{r}\right.$ $=0.1$ ). Thin and thick lines correspond to the finite and infinite wavelength instability.

less constant for many liquids and equal to $10^{-3} \mathrm{~K}^{-1}$, the value $10^{3} \delta_{\rho}$ reported on the vertical axis gives a direct estimate of the temperature drop (in ${ }^{\circ} \mathrm{C}$ ) across the liquid layer. We stress that only results which are compatible with the assumption $\delta_{\rho c}<0.1$ have been plotted. Various curves are given for $\Gamma$ ranging from $10^{-4}$ to $10^{2}$. For most liquids, these values correspond to liquid thicknesses ranging from about $0.1 \mathrm{~mm}$ to a few centimeters. Parameter $N$ takes values between $10^{-7}$ and $10^{-3}$, which are representative of viscosities ranging from $10^{-6}$ to $10^{-2} \mathrm{~m}^{2} / \mathrm{s}$ (from $10^{-2}$ to $10^{2} \mathrm{~S}$ ).

Some comments are in order about Fig. 3. First, the instability threshold for the surface zero-wave number mode, which is represented with a thick line, is independent of $N$. Indeed, this surface instability mode is independent of the viscosity of the liquid, since it is characterized by a vanishing velocity field. On the other hand, the thin lines in Fig. 3 correspond to the cellular mode of convection. The increase of the critical temperature difference with $N$ clearly illustrates the stabilizing role of viscosity.

The dependence of $\delta_{\rho c}$ with respect to $\Gamma$ deserves some attention too. First, a careful observation of the slope of the thick line in Fig. 3 shows that the critical temperature difference for the surface instability is nearly proportional to $\Gamma$, i.e., proportional to $d^{2}$. For the cellular instability, the slope of the curve is more or less equal to $-1 / 2$ for small values of $\Gamma$. When $\Gamma$ is increased, the slope progressively decreases to reach $-3 / 2$. From these values of the slope and from Eq. (3.4), it can be inferred that thermocapillarity is clearly the dominant instability motor for small values of $d$ while the Rayleigh buoyancy mechanism is the cause of convection in thick layers. For intermediate values of $d$, both mechanisms cooperate. Note also that for low viscosities, the instability is always thermocapillary driven since the slope of the curve remains equal to about $-1 / 2$. Similarly, the $\Gamma$ range for pure thermocapillary convection becomes very small for highly viscous liquids. 


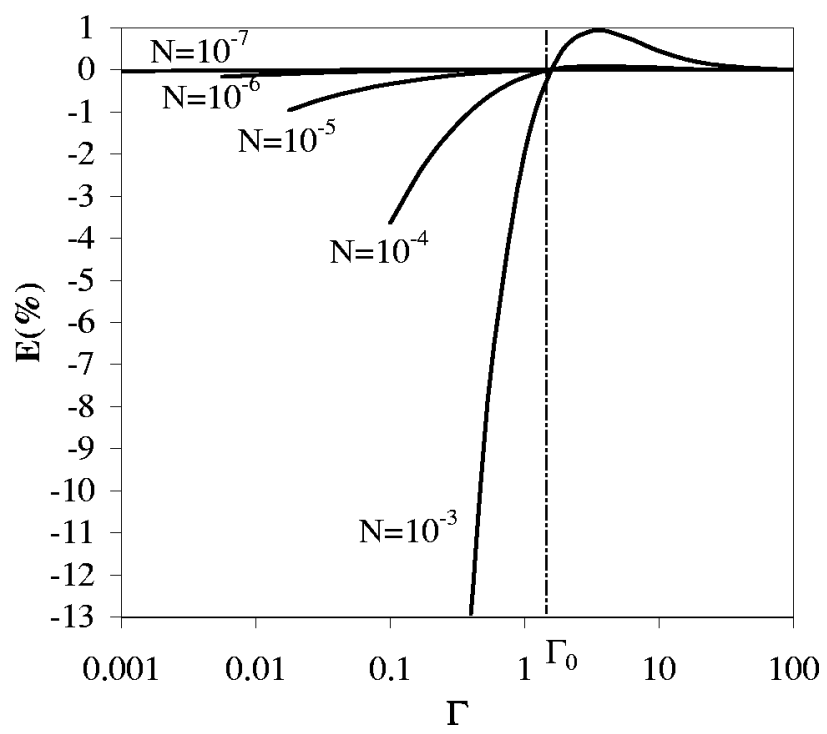

FIG. 4. $E$ vs $\Gamma$ for different values of $N\left(A=5, d_{r}=0.5, k_{r}=0.1\right)$.

The above-mentioned analysis is important because it enables one to determine the proper experimental conditions under which the surface instability with a zero wave number should be observed. Quite thin layers of very viscous fluid will favor this kind of instability. For instance, in silicone oil with viscosity $10^{-4} \mathrm{~m}^{2} / \mathrm{s}(1 \mathrm{St})$, the zero-wave number instability appears for a depth smaller than about $0.3 \mathrm{~mm}$. In a $10^{-3} \mathrm{~m}^{2} / \mathrm{s}(10 \mathrm{St})$ viscous oil, the critical thickness is about $1 \mathrm{~mm}$.

Similarly, one can also determine the most appropriate experimental conditions to observe "pure" Marangoni instability on earth. Low viscous liquids with small depths should be preferred but $d$ must be large enough to avoid the occurence of surface instability with a zero wave number. Under such circumstances, the critical temperature difference may, however, drop drastically (up to $10^{-1}{ }^{\circ} \mathrm{C}$ ) and is therefore delicate to control in experiments.

It is also possible to determine the importance of surface deformation on the finite wavelength instability by calculating the relative variation $E$ of the instability threshold with respect to the value corresponding to a flat surface:

$$
E=\frac{\delta_{\rho c}-\delta_{\rho c}(\eta=0)}{\delta_{\rho c}(\eta=0)} .
$$

We have reported $E$ in Fig. 4 as a function of $\Gamma$, for various values of $N$. It is seen that there exists a critical value $\Gamma_{0}$, independent of $N$, below which surface deformation destabilizes the system (e.g., $\Gamma_{0}=1.56$ when $d_{r}=0.5, k_{r}$ $=0.1$, and $A=5)$. If $\Gamma$ is larger than this critical value, deformations are stabilizing, in agreement with Ref. 37. A detailed study of the eigenfunctions shows that this critical value of $\Gamma$ corresponds to a change of sign of the curvature of the interface. ${ }^{4}$ When $\Gamma$ is larger than $\Gamma_{0}$, it is shown that the fluid is rising under the bumps of the upper surface. This configuration is characteristic of gravity-driven convection; in contrast, capillary thermoconvection is associated with small values of $\Gamma$ and with upwards motion under the troughs.
Figure 4 also indicates that the interface can reasonably be considered as nondeformed for low viscous fluids ( $N$ smaller than $10^{-5}-10^{-6}$ ). For more viscous liquids, deformation of the interface can modify the value of the convective threshold by several percent, especially in thin layers (small $\Gamma$ ), i.e., in the case of capillary convection. For gravity-driven convection (liquid depth of the order of $1 \mathrm{~cm}$ ), the influence of surface deformation remains always minute, whatever the value of the viscosity of the liquid.

\section{NONLINEAR ANALYSIS OF CELLULAR CONVECTION}

A linear stability analysis enables one to determine the critical wave number and the critical value of the control parameter above which convection sets in. In a nonlinear approach, the behavior of the system above the linear threshold is investigated. In order to clearly define the purpose of our work, it is interesting to refer to the classification introduced by Cross and Hohenberg ${ }^{38}$ of the different types of linear instability of a spatially uniform state. The type I instability is characterized by a nonzero wave number at the threshold and can be either stationary or oscillatory. In type II and type III instabilities, the critical wave number is zero at the threshold and the instability can also be stationary or oscillatory. In the nonlinear regime, the type II instability is characterized by the property that the zero wave number mode always keeps a vanishing growth rate while this quantity becomes positive for type III. In our problem, the instability can be either of type $\mathrm{I}_{s}$ or $\mathrm{II}_{s}$ (index $s$ means "stationary") depending on the values of the different parameters.

Several nonlinear analyses of thermoconvective instabilities with a deformable upper surface have already been published in the past. A pioneering work is that by Kraska and $\mathrm{Sani}^{39}$ where rolls and hexagonal cells have been studied. However, their approach was critized by Rosenblat et $a l .{ }^{40}$ and by Davis. ${ }^{41}$ The type $\mathrm{III}_{s}$ intability was analyzed by Golovin et al. in Ref. 42 and by Hadji in Ref. 43 while type $\mathrm{II}_{s}$ instability in thermocapillary convection was studied by Davis in Ref. 44, Funada and Kotani in Ref. 45, and by Funada in Ref. 46. Another interesting analysis of the competition between the $\mathrm{I}_{s}$ and $\mathrm{II}_{s}$ thermocapillary intabilities was also proposed by Golovin et al. in Ref. 47. In the present work, we concentrate on the study of the $\mathrm{I}_{s}$ cellular intability, characterized by a nonzero wave number. Both the thermocapillary and thermogravific mechanisms are taken into account and the upper gas layer is considered as purely conductive. Our approach can be seen as a correction and an extension of the work by Kraska and Sani, since besides the rolls and hexagonal cells, the square convective cells are also introduced in the analysis. The method used to derive the Ginzburg-Landau amplitude equations for rolls, square, and hexagonal convective patterns is completely similar to that presented in Ref. 15. These amplitude equations are written as

$$
\begin{aligned}
\tau \frac{d A_{1}}{d t}= & \epsilon A_{1}+a \overline{A_{2}} \overline{A_{3}}-b\left(\left|A_{2}\right|^{2}+\left|A_{3}\right|^{2}\right) A_{1}-c\left|A_{1}\right|^{2} A_{1} \\
& -d\left(\left|A_{5}\right|^{2}+\left|A_{6}\right|^{2}\right) A_{1}-e\left|A_{4}\right|^{2} A_{1},
\end{aligned}
$$




$$
\begin{aligned}
\tau \frac{d A_{2}}{d t}= & \epsilon A_{2}+a \overline{A_{1}} \overline{A_{3}}-b\left(\left|A_{1}\right|^{2}+\left|A_{3}\right|^{2}\right) A_{2}-c\left|A_{2}\right|^{2} A_{2} \\
& -d\left(\left|A_{4}\right|^{2}+\left|A_{6}\right|^{2}\right) A_{2}-e\left|A_{5}\right|^{2} A_{2}, \\
\tau \frac{d A_{3}}{d t}= & \epsilon A_{3}+a \overline{A_{1}} \overline{A_{2}}-b\left(\left|A_{1}\right|^{2}+\left|A_{2}\right|^{2}\right) A_{3}-c\left|A_{3}\right|^{2} A_{3} \\
& -d\left(\left|A_{4}\right|^{2}+\left|A_{5}\right|^{2}\right) A_{3}-e\left|A_{6}\right|^{2} A_{3}, \\
\tau \frac{d A_{4}}{d t}= & \epsilon A_{4}+a \overline{A_{5}} \overline{A_{6}}-b\left(\left|A_{5}\right|^{2}+\left|A_{6}\right|^{2}\right) A_{4}-c\left|A_{4}\right|^{2} A_{4} \\
& -d\left(\left|A_{2}\right|^{2}+\left|A_{3}\right|^{2}\right) A_{4}-e\left|A_{1}\right|^{2} A_{4}, \\
\tau \frac{d A_{5}}{d t}= & \epsilon A_{5}+a \overline{A_{4}} \overline{A_{6}}-b\left(\left|A_{4}\right|^{2}+\left|A_{6}\right|^{2}\right) A_{5}-c\left|A_{5}\right|^{2} A_{5} \\
& -d\left(\left|A_{1}\right|^{2}+\left|A_{3}\right|^{2}\right) A_{5}-e\left|A_{2}\right|^{2} A_{5}, \\
\tau \frac{d A_{6}}{d t}= & \epsilon A_{6}+a \overline{A_{4}} \overline{A_{5}}-b\left(\left|A_{4}\right|^{2}+\left|A_{5}\right|^{2}\right) A_{6}-c\left|A_{6}\right|^{2} A_{6} \\
& -d\left(\left|A_{1}\right|^{2}+\left|A_{2}\right|^{2}\right) A_{6}-e\left|A_{3}\right|^{2} A_{6} .
\end{aligned}
$$

In these equations, $A_{i}$ are the complex amplitudes of the six elementary convective modes whose superpositions allow one to generate roll-like, square, and hexagonal patterns ${ }^{15}$ while $\tau, a, b, c, d$, and $e$ are numbers whose values depend on the parameters of the problem.

Before going into the discussion of the results, let us mention that two new nondimensional quantities will be introduced in this section with respect to the linear analysis because the unsteady behavior of the system is now taken into account. One of these quantities is the ratio $\kappa_{r}$ of the thermal diffusivities of the gas and of the liquid. It follows from Table I that $\kappa_{r}$ is of the order of $10^{2}$ in most cases. Therefore, it will be kept fixed to that value in the following. The second relevant dimensionless number is

$$
F=\frac{\operatorname{Pr}}{N}=\frac{1}{\kappa^{2}}\left(\frac{\gamma^{3}}{\rho^{3} g \alpha^{3}}\right)^{1 / 2} .
$$

In contrast to the classical Prandtl number, $F$ does not depend on the viscosity. Thanks to this choice, the viscosity variations are taken into account by the sole parameter $N$. Table IV shows that $F$ ranges from $10^{6}$ to $10^{8}$. In the analysis of the results to follow, this quantity will be fixed to the typical value $10^{7}$. Like in the linear analysis, $A$ and $k_{r}$ are fixed to 5 and 0.1 , respectively.

\section{A. Stability domains of the different convective planforms}

As a first example, take the ratio $d_{r}$ of the thicknesses of the air and of the liquid equal to 0.25 and the viscosity parameter $N$ equal to $10^{-3}$ (so that $\operatorname{Pr}=F N=10^{4}$ ). In Figs. 5 and 6 , we have represented the stable convective patterns as functions of the relative distance to the threshold $\epsilon$ and of the thickness parameter $\Gamma$. The meaning of the different symbols is the following: $C=$ conductive state, $H^{+}=$upflow hexagonal cells, $R=$ rolls, $S=$ square cells. Dashed lines correspond to a flat interface while solid lines refer to a deformable

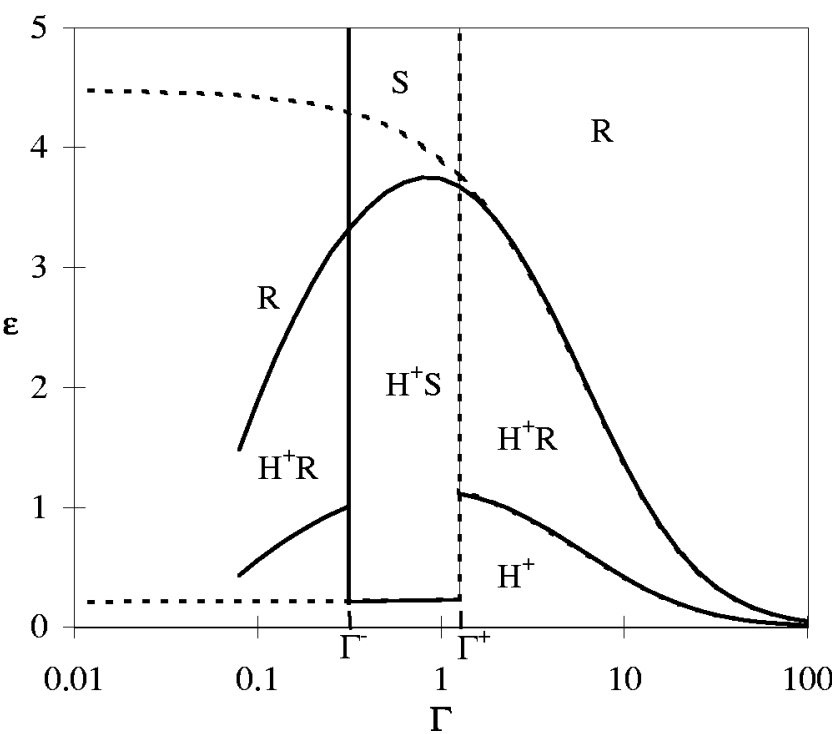

FIG. 5. Supercritical stability region vs $\Gamma$ for $F=10^{7}, N=10^{-3}$ (Pr $\left.=10^{4}\right), A=5, d_{r}=0.25, k_{r}=0.1, \kappa_{r}=10^{2} .\left(R, H^{+}\right.$, and $S$ correspond to rolls, upflow hexagons, and squares.) The dashed and solid lines describe an undeformable and a deformable interface, respectively.

gas-liquid surface. From Fig. 6, it is inferred that the bifurcation at the linear threshold is subcritical to upflow hexagons. For higher values of $\epsilon$, transitions to rolls or square cells are observed, with the important property that, for a given value of $\Gamma$, either rolls or squares are possible, but not both. Accordingly, two "critical" values of the thickness parameter, $\Gamma^{-}$and $\Gamma^{+}$, can be introduced which define an interval on the horizontal axis in which the transition to squares is observed. Another important conclusion from Figs. 5 and 6 is that deformations are relevant only for small values of $\Gamma$. For these values of the thickness parameter, the size of the stability region for hexagonal cells is reduced (for

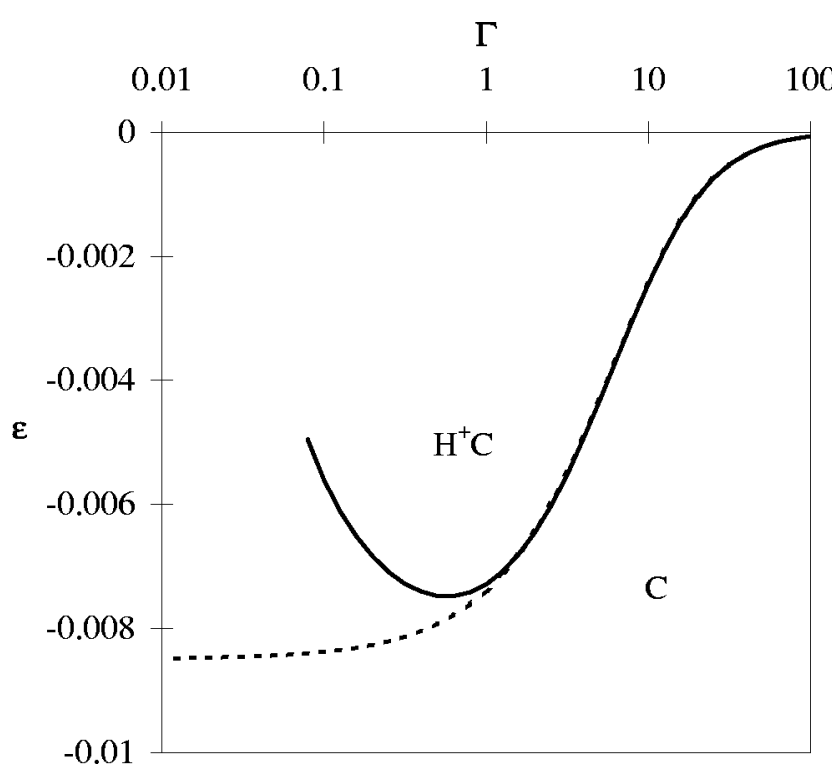

FIG. 6. Subcritical stability region vs $\Gamma$ for $F=10^{7}, N=10^{-4}\left(\operatorname{Pr}=10^{3}\right)$, $A=5, d_{r}=0.25, k_{r}=0.1, \kappa_{r}=10^{2} .\left(H^{+}\right.$and $C$ stand for upflow hexagons and the conductive state, respectively.) The dashed line refers to an undeformable interface, the solid one to a deformable interface. 


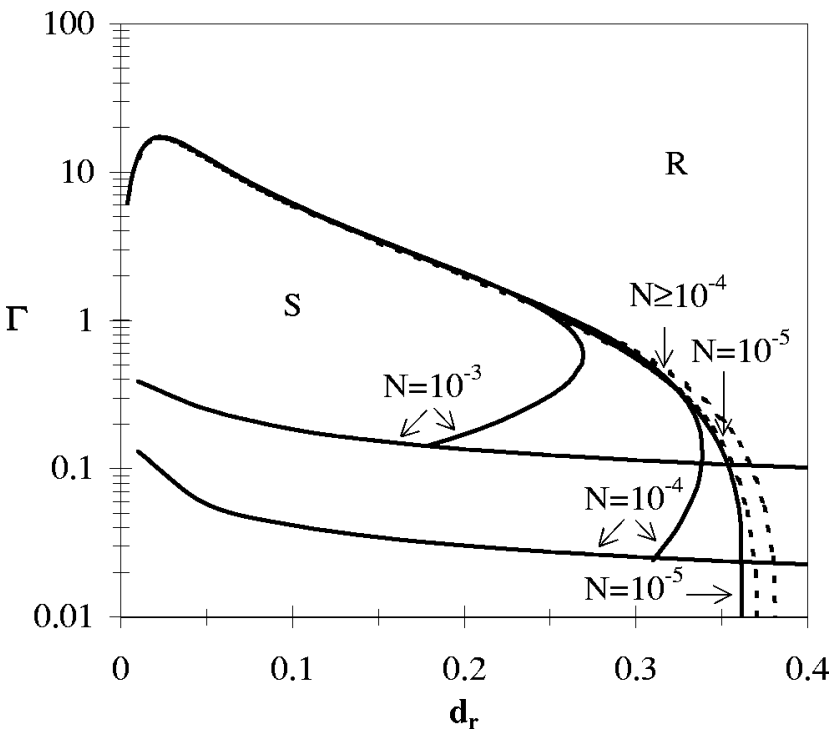

FIG. 7. Stability domains in the $\Gamma-d_{r}$ plane for rolls $(R)$ and square cells $(S)$ beyond the second bifurcation and for different values of the viscosity parameter $N\left(F=10^{7}, A=0, k_{r}=0.1, \kappa_{r}=10^{2}\right)$. Thin and thick lines correspond to the finite and infinite wavelength instability; dashed lines refer to the nondeformable interface.

both the supercritical and subcritical regime) with respect to the flat surface case. Note also that the full lines end at a value of $\Gamma$ below which the threshold is charaterized by a zero wave number.

When different values for $d_{r}$ and $N$ are selected, the following conclusions can be drawn. First, for values of the viscosity parameter $N$ smaller than $10^{-5}\left(\operatorname{Pr}<10^{2}\right)$, the surface deflection can be neglected; the corresponding results were given in Ref. 15 and will not be commented on further. For higher values of $N$, which are the only values considered in the following, our analysis has shown that the primary bifurcation is still subcritical to upflow hexagonal cells (when the zero wave-number mode is stable). However, the stability domain for hexagons is reduced with respect to the flat interface problem, by a factor which increases up to 2 when the viscosity parameter is increased to the value $N$ $=10^{-3}$. A summary of the conditions giving rise to a secondary transition to square or to roll-like cells is also presented in Fig. 7, with dashed and full thin lines for flat and deformable interface, respectively. The full thick lines correspond to the zero wave-number instability. Consider first the nondeformable interface. From Fig. 7, it is seen that for fixed $d_{r}$ and $N$, a critical $\Gamma^{+}$exists above which only rolls appear. In contrast with the above-studied case (Figs. 5 and 6), no critical $\Gamma^{-}$exists so that the $\Gamma$ domain for which a transition to squares exists stretches from 0 to $\Gamma^{+}$. We can also note that the critical $\Gamma^{+}$is almost unchanged when $N$ is increased from $10^{-5}$ and that it is almost always a decreasing function of $d_{r}$. When deformations are taken into account, the situation is quite different. Indeed, when the viscosity parameter is increased from $10^{-5}$, the stability domain for square cells is reduced because of the appearance of the zero wave number instability and also because the curve showing the boarder between $R$ and $S$ bends to give birth to a $\Gamma^{+}$and a $\Gamma^{-}$critical value. In particular, it is worth mentioning that

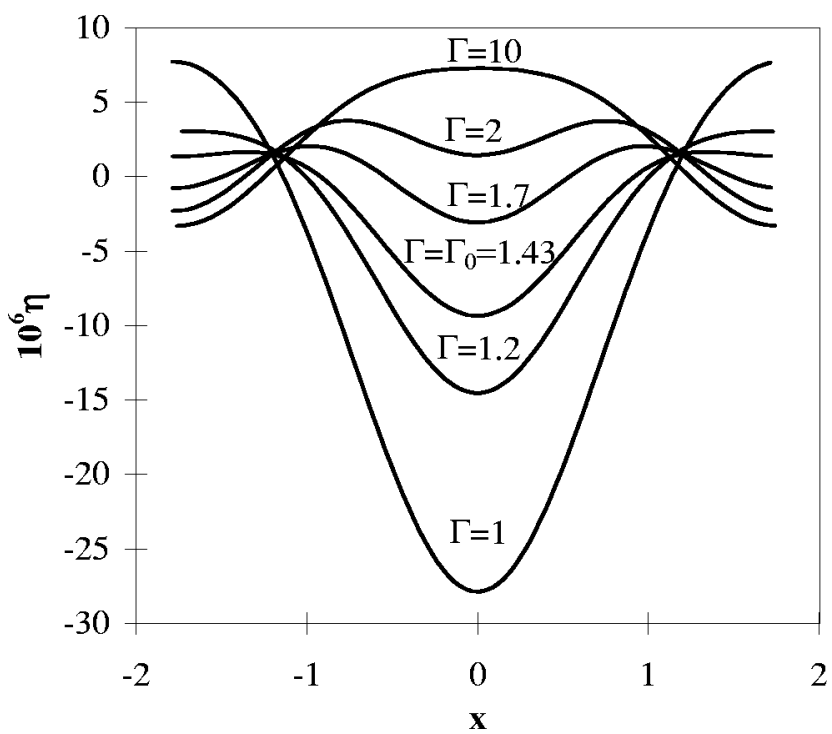

FIG. 8. Shape of the interface in a vertical plane crossing the middle of two opposite sides of a hexagonal cell for $\epsilon=10^{-1}$ and for different values of $\Gamma$ $\left[F=10^{7}, N=10^{-4}\left(\operatorname{Pr}=10^{3}\right), A=5, d_{r}=0.25, k_{r}=0.1, \kappa_{r}=10^{2}\right]$.

secondary transitions to roll-like cells in thin layers can only occur in the case of a deformed interface.

Before closing this section, let us mention that some interesting experimental results have been published recently by Eckert et al. ${ }^{16}$ In particular, these authors found that a secondary instability, from hexagonal cells to square cells, occurs at $\epsilon=4.2 \pm 0.3$ for $\mathrm{Pr}=100$ while our model predicts a transition for $\epsilon=4.05$. This surprising quantitative agreement with experiments shows that amplitude equations, which are theoretically valid in the weakly nonlinear regime only, can sometimes provide good approximations even far from the threshold.

\section{B. Interface relief}

A further interest of a nonlinear analysis is the possibility to study in detail the shape of the interface and to determine the amplitude of the surface deflections in order to compare the theoretical predictions with the experimental results available in the work by Cerisier et al. ${ }^{17}$

When $\Gamma$ is different from the threshold value $\Gamma_{0}$ introduced in Sec. III B 3, a linear stability analysis provides some partial information on the shape of the interface but the amplitude of the deformation cannot be determined. When $\Gamma$ is close to $\Gamma_{0}$, the linear surface deformation vanishes and only a nonlinear analysis enables one to predict both the form of the interface and the amplitude of the deformations. To compare with the experimental results of Cerisier et al., ${ }^{17}$ we restrict our analysis to $\mathrm{H}^{+}$solutions. In Fig. 8, we plotted for $\epsilon=0.1$ the surface deflections in a vertical plane crossing the middle of two opposite sides of the hexagon for different values of $\Gamma$. The viscosity parameter $N$ is taken equal to $10^{-4}$ (the corresponding Prandtl number is $10^{3}$, as in the experiments of Cerisier et al.) and $d_{r}=0.25$. For these values of the parameters, $\Gamma_{0}=1.43$. When $\Gamma$ is much larger than this value (buoyancy convection), a bump is predicted in the middle of the cell while a trough is displayed for small 


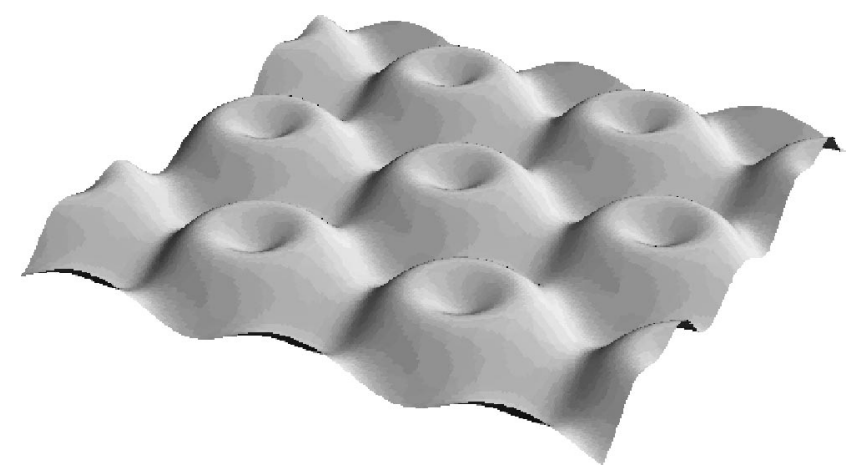

FIG. 9. Nonlinear "hybrid" deformations of the upper surface of hexagonal cells when $\Gamma$ is close to $\Gamma_{0}$.

thicknesses (thermocapillary effect). When $\Gamma$ is close to the critical value, the surface is no longer flat as predicted by the linear theory because the nonlinear harmonics of the fundamental hexagonal pattern, which are characterized by a nonvanishing surface deformation, have a nonzero amplitude. A general view of the form of the interface in this case ("hybrid" case) is represented in Fig. 9 which is very similar to the experimental observation of Cerisier et al. (see Fig. 5 in Ref. 17).

As a last check of the nonlinear model, we have calculated the amplitude of surface deformations and confronted our results with experiments. The comparison is presented in Fig. 10 for a layer of silicon oil 47V100 with thickness between 1.0 and $4.5 \mathrm{~mm}$. The relative distance to the linear threshold $\epsilon$ is equal to 0.5 . The dimensionless difference in height between the side and the center of the cells, $H_{b}$, is represented as a function of $\Gamma$. Since no lid was used in the experiments of Cerisier et al. to delimit precisely the air layer, we can consider that the agreement between theory (solid lines) and experiment (dots) is quite satisfactory, especially for thick layers.

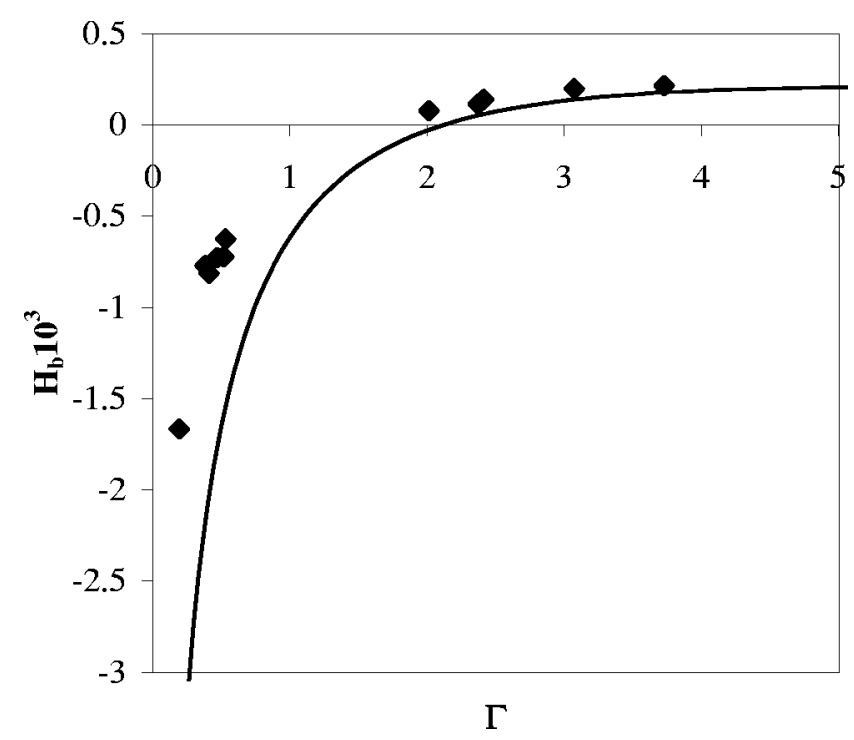

FIG. 10. Comparison between the experimental (dots) and theoretical (solid line) amplitudes of the surface deformation vs $\Gamma$.

\section{CONCLUSION}

A linear and nonlinear analysis of Rayleigh-BénardMarangoni instability with surface deformations is presented in this paper.

The linear approach is carried out in two steps. First, the role of the upper gas layer in Rayleigh-Bénard-Marangoni instability is examined in detail and the frequently used assumption of a purely conductive gas is proved to be valid when the thickness of the gas layer is smaller than that of the liquid.

The second step of the linear part of the work consists in studying the influence of surface deformations when the upper gas can be considered as purely conductive. The simplified Oberbeck-Boussinesq model is used and its validity is carefully analyzed. In particular, we show that consistency is achieved provided $\delta_{\rho} \ll 1$ [see Eq. (2.3)].

The most important results from the linear study are the following. First, it is concluded that oscillatory convection is not possible under realistic experimental conditions with $\delta_{\rho}$ $\ll 1$. Besides, it was shown that, among the relatively large number of parameters appearing in the problem, the viscosity, measured by the new nondimensional number $N$, is by far the most important. Indeed, the other nondimensional numbers characterizing the thermophysical properties of the fluids keep a more or less constant value for a large class of liquids generally used in experiments on thermoconvection. The general stability results are summarized in Fig. 3 wherein the convection thresholds are plotted as a function of the parameter $\Gamma$ (the dimensionless measure of the liquid depth), for various values of the viscosity parameter $N$. The experimental conditions required to observe the surface zero wave-number instability can also be deduced from this picture, as well as the conditions under which gravity effects may be neglected in thermoconvective motions on earth.

We have also determined under which circumstances the interface can be considered as remaining flat. Figure 4 indicates that surface deformations can always be neglected in low viscous fluids. In contrast, these deformations become important in more viscous liquids, especially in thin layers where the thermocapillary instability is dominating.

As a side contribution, we have introduced four new dimensionless groups. The nondimensional form of temperature differences is represented by means of the abovementioned parameter $\delta_{\rho}$. Recall once again the importance of this quantity in relation to the validity of the Boussinesq approximation. Parameter $A$ is related to the surface tension while $\Gamma$ is a measure of the thickness of the liquid layer as well as of the relative importance of gravity and thermocapillarity in the instability mechanism. Finally, the viscosity of the fluid is described by a fourth parameter $N$.

In the nonlinear part of the paper, the behavior of the fluid above the threshold is examined, with the assumption of a conductive upper gas. More precisely, the finite wavelength convection is studied and the competition between roll-like, square and hexagonal cells is investigated. An interesting conclusion of the analysis is that the $\epsilon$ domain wherein hexagonal cells are stable is smaller in the presence of surface deformations. This effect is still accentuated when 
the viscosity is increased or when the thickness is decreased. Another important result is the transition to rolls or to square cells when the temperature gradient is increased. When the surface is flat, roll-like and square cells appear, respectively, in thick and thin layers, with a critical value $\Gamma^{+}$of the thickness parameter that defines the limit between both cases. In contrast, with a deformable interface and for rather viscous liquids, square convective cells are possible for values of $\Gamma$ contained between two limits, $\Gamma^{-}$and $\Gamma^{+}$. As a consequence, rolls can also be observed in sufficiently thin layers.

Our theoretical analysis was also compared with some experimental data by Cerisier et al. ${ }^{17}$ on interface deformations. In particular, it was shown that the observed "hybrid", relief of the interface originates in the nonlinear influence of harmonics of the linearly unstable solution. We have also shown that the amplitude of the deformations calculated with our model is in satisfactory agreement with the experimental data. Eventually, the calculated value of $\epsilon$ corresponding to the transition between hexagonal and square cells was shown to be in good agreement with the experimental value reported by Eckert et al. ${ }^{16}$

\section{ACKNOWLEDGMENTS}

This text presents results of the Belgian InterUniversity Pole of Attraction (IUPA) under Contract No. IV.06. Fruitful discussions with Professor J.-C. Legros (Brussels Free University), Professor J. K. Platten (Mons University) and their groups are acknowledged.

${ }^{1}$ E. L. Koschmieder, Bénard Cells and Taylor Vortices (Cambridge University Press, Cambridge, 1993).

${ }^{2}$ S. Rasenat, F. H. Busse, and I. Rehberg, "A theoretical and experimental study of double-layer convection," J. Fluid Mech. 199, 519 (1989).

${ }^{3}$ D. Johnson, R. Narayanan, and P. C. Dauby, "The effect of air height on the pattern formation in liquid-air bilayer convection," in Fluids Dynamics at Interfaces, edited by W. Shyy and R. Narayanan (Cambridge University Press, Cambridge, 1999).

${ }^{4}$ L. E. Scriven and C. V. Sterling, "On cellular convection driven by surface-tension gradients: Effects of mean surface tension and surface viscosity," J. Fluid Mech. 19, 321 (1964).

${ }^{5}$ A. Smith, "On convective instability induced by surface-tension gradients," J. Fluid Mech. 24, 401 (1966).

${ }^{6}$ R. W. Zeren and W. C. Reynolds, "Thermal instabilities in two-fluid horizontal layers," J. Fluid Mech. 53, 305 (1972).

${ }^{7}$ M. Takashima, "Surface tension driven instability in a horizontal liquid layer with a deformable free surface. I. Stationary convection," J. Phys. Soc. Jpn. 50, 2745 (1981).

${ }^{8}$ M. Takashima, "Surface tension driven instability in a horizontal liquid layer with a deformable free surface. II. Overstability," J. Phys. Soc. Jpn. 50, 2751 (1981).

${ }^{9}$ J. Reichenbach and H. Linde, "Linear perturbation analysis of surfacetension-driven convection at a plane interface," J. Colloid Interface Sci. 84, 433 (1981).

${ }^{10}$ E. N. Ferm and D. J. Wolkind, "Onset of R-B-M instability: Comparison between theory and experiment," J. Non-Equilib. Thermodyn. 7, 169 (1982).

${ }^{11}$ D. A. Goussis and R. E. Kelly, "On the thermocapillary instabilities in a liquid layer heated from below," Int. J. Heat Mass Transf. 33, 2237 (1990).

${ }^{12}$ G. Gouesbet, J. Maquet, C. Rozé, and R. Darrigo, "Surface-tension and coupled buoyancy-driven instability in a horizontal liquid layer. Overstability and exchange of stability," Phys. Fluids A 2, 903 (1990).

${ }^{13}$ C. Pérez Garcia and G. Carneiro, "Linear stability analysis of B-M convection in fluids with a deformable free surface," Phys. Fluids A 3, 292 (1991).

${ }^{14}$ Y. Renardy and M. Renardy, "Bifurcating solutions at the onset of con- vection in the B problem for two fluids,"' Physica D 32, 227 (1988).

${ }^{15}$ V. C. Regnier, P. M. Parmentier, P. C. Dauby, and G. Lebon, "'Square cells in gravitational and capillary thermoconvection," Phys. Rev. E 55, 6860 (1997).

${ }^{16} \mathrm{~K}$. Eckert, M. Bestehorn, and A. Thess, "Square cells in surface-tensiondriven Bénard convection: Experiment and theory," J. Fluid Mech. 356, 155 (1998).

${ }^{17}$ P. Cerisier, C. Jamond, J. Pantaloni, and J. C. Charmet, "Déformation de la surface libre en convection de Bénard-Marangoni," J. Phys. 45, 405 (1984).

${ }^{18}$ I. B. Simanovskii and A. A. Nepomnyashchy, Convective Instabilities in Systems with Interface (Gordon and Breach, Amsterdam, 1993).

${ }^{19}$ D. D. Joseph, Stability of Fluid Motion I/II (Springer, Berlin, 1976).

${ }^{20}$ R. Sélak and G. Lebon, "Bénard-Marangoni thermoconvective instability in presence of a temperature-dependent viscosity," J. Phys. 3, 1185 (1993).

${ }^{21}$ R. Sélak and G. Lebon, "Rayleigh-Marangoni thermoconvective instability with non-Boussinesq corrections," Int. J. Heat Mass Transf. 40, 785 (1996).

${ }^{22}$ P. G. Drazin and W. H. Reid, Hydrodynamic Stability (Cambridge University Press, Cambridge, 1981).

${ }^{23}$ V. C. Regnier, "Instabilités thermoconvectives dans les fluides à interfaces déformables," Ph.D. thesis, Université de Liège, 1998.

${ }^{24}$ E. L. Koschmieder, "'On convection under an air surface,'” J. Fluid Mech. 30, 9 (1967).

${ }^{25} \mathrm{H}$. Jeffreys, "Some cases of instability in fluid motion," Proc. R. Soc. London, Ser. A 118, 195 (1928).

${ }^{26} \mathrm{D}$. A. Nield, "Surface tension and buoyancy effects in cellular convection," J. Fluid Mech. 9, 341 (1964).

${ }^{27}$ S. J. Vanhook, M. F. Schatz, J. B. Swift, W. D. McCormick, and H. L. Swinney, "Long-wavelength surface-tension-driven Bénard convection: Experiment and theory,"' J. Fluid Mech. 345, 45 (1997).

${ }^{28}$ V. C. Regnier and G. Lebon, "Time-growth and correlation length of fluctuations in thermocapillary convection with surface deformation," $\mathrm{Q}$. J. Mech. Appl. Math. 48, 57 (1995).

${ }^{29} \mathrm{C}$. Normand, Y. Pomeau, and M. Velarde, "Convective instability: A physicist's approach,' Rev. Mod. Phys. 49, 581 (1977).

${ }^{30}$ C. Pérez-García, B. Echebarría, and M. Bestehorn, "Thermal properties in surface-tension-driven convection,’' Phys. Rev. E 57, 475 (1998).

${ }^{31}$ E. L. Koschmieder and S. A. Prahl, "Surface-tension-driven Bénard convection in small containers," J. Fluid Mech. 215, 571 (1990).

${ }^{32}$ J. Pearson, "On convection cells induced by surface tension," J. Fluid Mech. 4, 489 (1958).

${ }^{33} \mathrm{~L}$. Segel, "The nonlinear interaction of a finite number of disturbances to a layer of fluid heated from below,"' J. Fluid Mech. 21, 359 (1965).

${ }^{34}$ A. Cloot and G. Lebon, "A nonlinear stability analysis of the BénardMarangoni problem,'” J. Fluid Mech. 145, 447 (1984).

${ }^{35}$ P. Parmentier, V. C. Regnier, G. Lebon, and J. C. Legros, "A nonlinear analysis of coupled gravitational and capillary thermoconvection in thin fluid layers,"' Phys. Rev. E 54, 411 (1996).

${ }^{36}$ P. C. Dauby and G. Lebon, "Bénard-Marangoni instability in rigid rectangular containers," J. Fluid Mech. 329, 25 (1996).

${ }^{37}$ S. H. Davis and G. M. Homsy, "Energy stability theory for free-surface problems: Buoyancy-thermocapillary layers," J. Fluid Mech. 98, 527 (1980).

${ }^{38}$ M. Cross and P. Hohenberg, "Pattern formation outside of equilibrium," Rev. Mod. Phys. 5, 851 (1993).

${ }^{39}$ J. Kraska and R. Sani, "Finite amplitude Bénard-Rayleigh convection," Int. J. Heat Mass Transf. 22, 535 (1979).

${ }^{40}$ S. Rosenblat, S. H. Davis, and G. M. Homsy, "Nonlinear Marangoni convection in bounded layers. 1. Circular cylindrical containers,' J. Fluid Mech. 120, 91 (1982).

${ }^{41}$ S. H. Davis, “Thermocapillary instabilities," Annu. Rev. Fluid Mech. 19, 403 (1987).

${ }^{42}$ A. Golovin, A. Nepomnyashchy, and L. Pismen, "Pattern formation in large-scale Marangoni convection with deformable surface," Physica D 81, 117 (1995).

${ }^{43} \mathrm{~L}$. Hadji, "Nonlinear analysis of the coupling between interface deflection and hexagonal patterns in Rayleigh-Bénard-Marangoni convection," Phys. Rev. E 53, 5982 (1996).

${ }^{44}$ S. H. Davis, Rupture of Thin Liquids Films, Waves on Fluid Interfaces (Academic, New York, 1983). 
${ }^{45}$ T. Funada and M. Kotani, "A numerical diffusion equation governing surface deformation in the Marangoni convection,', J. Phys. Soc. Jpn. 55, 3857 (1986)

${ }^{46} \mathrm{~T}$. Funada, "Nonlinear surface waves driven by the Marangoni instability in a heat transfer system,'’ J. Phys. Soc. Jpn. 56, 2031 (1987).

${ }^{47}$ A. Golovin, A. Nepomnyashchy, and L. Pismen, "Interaction between short-scale Marangoni convection and long-scale deformational instability,' Phys. Fluids 6, 34 (1994). 
Physics of Fluids is copyrighted by the American Institute of Physics (AIP). Redistribution of journal material is subject to the AIP online journal license and/or AIP copyright. For more information, see http:/ojps.aip.org/phf/phfcr.jsp

Copyright of Physics of Fluids is the property of American Institute of Physics and its content may not be copied or emailed to multiple sites or posted to a listserv without the copyright

holder's express written permission. However, users may print, download, or email articles for individual use. 